\title{
High-responsivity plasmonics-based GaAs metal-semiconductor-metal photodetectors
}

\author{
Ayman Karar, ${ }^{1, a)}$ Narottam Das, ${ }^{1, b)}$ Chee Leong Tan, ${ }^{2}$ Kamal Alameh, ${ }^{1,3,4, c)}$ \\ Yong Tak Lee, ${ }^{2,3,4}$ and Fouad Karouta ${ }^{5}$ \\ ${ }^{1}$ Electron Science Research Institute, Edith Cowan University, Joondalup, WA, Australia \\ ${ }^{2}$ School of Photonics Science, Gwangju Institute of Science and Technology (GIST), Gwangju, South Korea \\ ${ }^{3}$ Department of Information and Communications, GIST, Gwangju, South Korea \\ ${ }^{4}$ Department of Nanobio Materials and Electronics, GIST, Gwangju, South Korea \\ ${ }^{5}$ ANFF, Research School of Physics \& Engineering, Australian National University, Canberra, Australia
}

(Received 4 April 2011; accepted 20 July 2011; published online 28 September 2011)

\begin{abstract}
We report the experimental characterization of high-responsivity plasmonics-based GaAs metal-semiconductor-metal photodetector (MSM-PD) employing metal nano-gratings. Both the geometry and light absorption near the designed wavelength are theoretically and experimentally investigated. The measured photocurrent enhancement is 4-times in comparison with a conventional single-slit MSM-PD. We observe reduction in the responsivity as the bias voltage increases and the input light polarization varies. Our experimental results demonstrate the feasibility of developing a high-responsivity, low bias-voltage high-speed MSM-PD. (C) 2011 American Institute of Physics. [doi:10.1063/1.3625937]
\end{abstract}

Metal-semiconductor-metal photodetectors (MSM-PDs) are very attractive devices due to their potential applications in optical fiber communication systems, high-speed chip-tochip interconnects, and high-speed sampling. ${ }^{1,2}$ Due to their lateral geometry, MSM-PDs have much smaller capacitance per unit area in comparison to standard p-i-n photodiode with same active area. The surface reflectivity and the shadowing due to the metal fingers prevent an ideal MSM-PD from achieving external quantum efficiency greater than $50 \%$ for equal electrode width and spacing. On the other hand, the smaller finger width, the less detector capacitance, and the shorter external response time. ${ }^{3}$ By decreasing the spacing between the electrode fingers down to the optical diffraction limit, the response times could be in the range of a few tens of picoseconds. ${ }^{4}$ However, this downsizing of the electrode spacing decreases the active area, thus resulting in photodetector sensitivity degradation.

Since the extraordinary optical transmission (EOT) phenomenon, which was reported by Ebbesen et al., ${ }^{5}$ many efforts have been devoted to exploring the EOT through metallic gratings with various sub-wavelength structures, such as periodic slit arrays, hole arrays, and corrugated metal films for different wavelength regions. ${ }^{6-8}$ It has also been established that the transmission of light through a hole or sub-wavelength slit in a metal film can be enhanced by micro-structuring the top or bottom surfaces of the film with gratings. These gratings couple the incident light with the surface plasmon polaritons (SPPs), which are guided into the sub-wavelength slit. ${ }^{11}$

Several different implementations of SPP-enhanced MSM-PDs have been reported confirming that the confinement of light in sub-wavelength metal-semiconductor nano-

\footnotetext{
${ }^{a)}$ Electronic mail: a.karar@ecu.edu.au. Telphone: $+61 \quad 8 \quad 63045836$. FAX: +61 863045302 .

${ }^{b}$ Present address: Department of Electrical and Computer Engineering, Curtin University, WA, Australia.

${ }^{c)}$ Electronic mail: k.alameh@ecu.edu.au.
}

gratings can be achieved by Fabry-Pérot resonances involving vertical transverse magnetic (TM) and electric guided waves, resulting in increasing the quantum efficiency. ${ }^{12,13}$ Lee et al., ${ }^{14}$ have reported a plasmonic metal photonic crystal (MPC) integrated on a quantum dot infrared photodetector demonstrating more than 2-times enhancement in detectivity. Recently, we reported a semi-analytical model of light absorption around $830 \mathrm{~nm}$ for MSM-PDs with plasmonic nano-gratings using the finite difference time domain (FDTD) numerical method. ${ }^{17}$ In that report, we showed that the transmission enhancement strongly depends on several parameters of the device structure, such as, the shape, height, and grating period, as well as, the sub-wavelength aperture width.

In this letter, we report on the development of a plasmonics-based GaAs MSM-PD structure fabricated using focused ion beam (FIB) milling and demonstrate responsivity enhancement of 4-times in comparison with conventional single-slit MSM-PDs, attributed to the SPP-assisted extraordinary optical transmission through the metal nano-gratings.

Figure 1 illustrates the schematic diagram of the plasmonics-based MSM-PD structure. The sub-wavelength slit $\left(\chi_{\mathbf{W}}\right)$ is etched between the metal nano-gratings of a grating period $(\boldsymbol{\Lambda})$. The incident electromagnetic field is assumed a TM polarized wave with magnetic field oscillating in the direction parallel to the nano-grating grooves. The metallic sub-wavelength aperture supports a propagating TM mode with the EOT. Consequently, with an appropriate choice of its width, the sub-wavelength aperture forms a Fabry-Pérot resonator; therefore, the light transmission through the subwavelength aperture is resonantly enhanced. On the other hand, the nano-gratings enhance the light transmission through the sub-wavelength aperture region by converting the incident electromagnetic (EM) waves into SPPs propagating on the metallic surface, which can be funnelled into the sub-wavelength aperture. With an accurate choice of geometric parameters of the structure, these two mechanisms can occur at the same time, resulting in an increase in the 


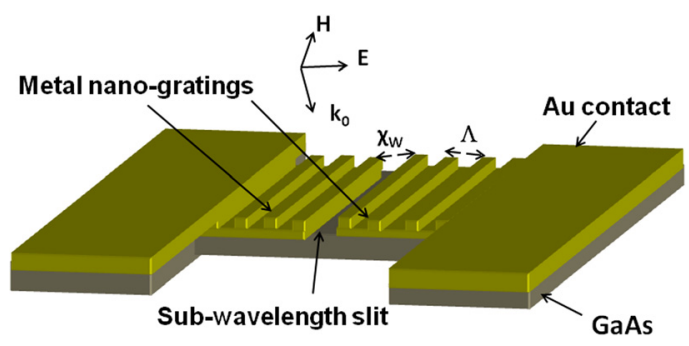

FIG. 1. (Color online) Schematic diagram of the plasmonic MSM-PD structure.

electron-hole pair generation rate and leading to a substantial increase in the overall photodetector responsivity.

The metal nano-gratings etched on both sides of the slit allow, through momentum difference, collective resonant coupling between the incident light and oscillating electrons. ${ }^{7}$ The metal nano-grating period $\boldsymbol{\Lambda}$ is obtained using the following dispersion relation ${ }^{9}$

$$
k_{\|}=k_{0} \sin \theta \pm \frac{2 n \pi}{\Lambda}=k_{0} \sqrt{\frac{\varepsilon_{m}^{\prime} \varepsilon_{d}}{\varepsilon_{m}^{\prime}+\varepsilon_{d}}}=k_{s p},
$$

where, $k_{\|}, k_{s p}$, and $k_{0}$ represent the parallel component of the incident light wave vector with respect to the device surface, the SPPs and free space wave vectors, respectively, $\boldsymbol{n}$ is an integer number and $\theta$ is the incident angle of the light. The complex dielectric permittivity of the metal is $\varepsilon_{m}=\varepsilon_{m}^{\prime}+i \varepsilon_{m}^{\prime \prime}$ and that of the air is $\boldsymbol{\varepsilon}_{\mathbf{d}}$. Using this relation, a period of $\boldsymbol{\Lambda}=815$ $\mathrm{nm}$ for the $\mathrm{Au} / \mathrm{air}$ interface results in an absorption spectrum of absorption edge around $830 \mathrm{~nm}$ for normal light incidence.

A thorough analysis of the plasmonics-based MSM-PD structure was performed using the two-dimensional FDTD method. A plane Gaussian-modulated continuous wave with TM polarization was used as an excitation source, as reported in Ref. 15. By simulating the electric flux transmitted into the active area for the same device with and without the nano-gratings, the light transmission enhancement factor $(\Gamma)$, defined as the ratio of the transmitted optical power with the nanogratings to that without nano-gratings, was easily calculated.

Figure 2(a) shows the light transmission enhancement factor versus wavelength for $200 \mathrm{~nm}, 250 \mathrm{~nm}$, and $300 \mathrm{~nm}$ slit widths. The grating period, grating height, and grooveto-pitch ratio were kept constant at $815 \mathrm{~nm}, 50 \mathrm{~nm}$, and 0.5 , respectively. These simulation results show that the light enhancement factor decreases rapidly with increasing the aperture width. For a slit width of $300 \mathrm{~nm}$, the light transmission enhancement factor is more than 6-times. Although the peak wavelength is shifted to $900 \mathrm{~nm}$, more than 3-times enhancement in light absorption is still attained at $830 \mathrm{~nm}$. Density plots of the electric and magnetic field components $\left(\mathbf{E}_{\mathbf{x}}, \mathbf{H}_{\mathbf{y}}\right.$, and $\left.\mathbf{E}_{\mathbf{z}}\right)$ without and with metal nano-gratings are shown in Fig. 2(b). The EM field distributions clearly reveal that with the incorporation of the nano-gratings, SPPs enhance the light transmission through the sub-wavelength aperture; much higher EM fields are induced the active area of the plasmonics-based MSM-PD device than those induced in a conventional MSM-PD device.

The fabrication process consisted of three steps. Initially, a stack of titanium/gold (Au/Ti 10:100nm) layers was sputtered (using an RF magnetron sputtering system) on top (a)
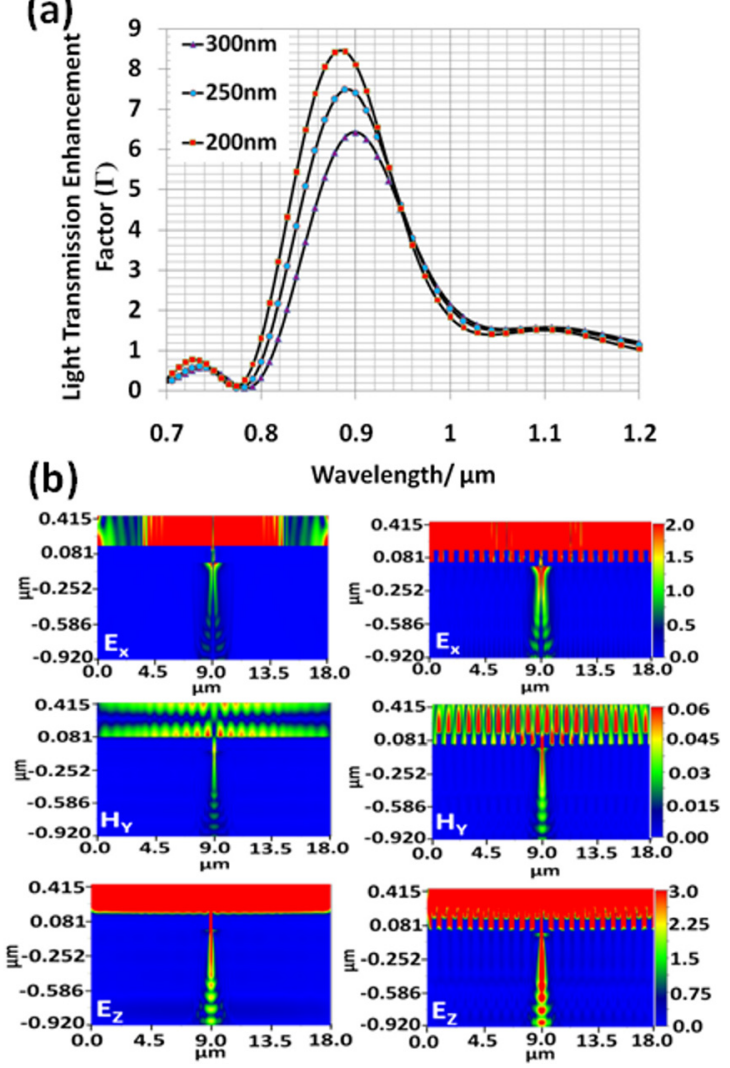

FIG. 2. (Color online) Simulated (a) light transmission enhancement factor $(\Gamma)$ versus wavelength and (b) field distributions $\left(E_{x}, H_{y}\right.$, and $\left.E_{z}\right)$ of the optimized MSM-PD device without (left) and with (right) nano-gratings.

of a semi-insulating GaAs substrate. Then, the top Au film was patterned using an FEI dual-beam FIB driven with a 10 pA $\mathrm{Ga}^{+}$ion beam current. Finally, $\mathrm{O}_{2}$ plasma etching and acid cleaning were used to remove the $\mathrm{Ga}^{+}$ion implanted onto the GaAs substrate surface within the slit. Figure 3 shows a $45^{\circ}$ tilted SEM image of the $230 \mathrm{~nm}$ slit MSM-PD with 10 nano-corrugation pitches being milled at each side. The inset of Fig. 3 shows a magnified image of the fabricated device, revealing a slit width of $230 \mathrm{~nm}$ and a grating period of around $830 \mathrm{~nm}$. A polarized $830 \mathrm{~nm}$ laser beam was focused to illuminate at the center of the active area of the MSM-PD, and the I-V characteristics for different input laser power levels were measured using an Agilent $4156 \mathrm{C}$ precision semiconductor parameter analyzer.

Figure 4(a) shows the measured I-V characteristics for both MSM-PDs (with and without nano-gratings) for two laser power levels ( 2 and $3 \mathrm{~mW}$ ). It is clear that the plasmonics-based MSM-PD structure (denoted as WG) produces a higher photocurrent in comparison to the single-slit MSMPD without nano-gratings (denoted as WOG). Figure 4(b) shows the dependency of the photocurrent enhancement factor (defined as the ratio between the current of the MSM-PD with a nano-grating $\left(\mathrm{I}_{\mathrm{WG}}\right)$ to the one without nano-grating ( $\mathrm{I}_{\mathrm{WOG}}$ ) for the same applied voltage) for an incident power of $3 \mathrm{~mW}$. For an optimum bias voltage of $0.5 \mathrm{~V}$, 4-times enhancement in photodetector responsivity is experimentally demonstrated. As seen in Fig. 4(b), at a high bias voltage, the photocurrent enhancement factor drops rapidly due to breakdown effects, where the electron-hole generation becomes less dependent on the incident light power. Note 


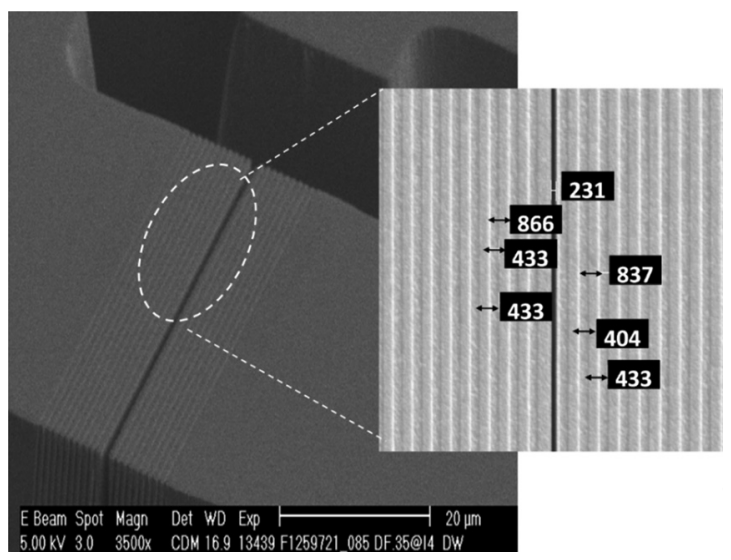

FIG. 3. An SEM image of the fabricated plasmonics-based MSM-PD structure. The inset shows a high magnification image of the sub-wavelength slit with linear nano-gratings at both sides.

that, since the plasmonics-based MSM-PD is a polarizationsensitive device, a polarization controller was used to ensure that the device was always illuminated by TM polarized light and maximum enhancement was attained for all the measurements.

As reported by Ren et al., ${ }^{13}$ in addition to the significant transmission enhancement, both high speed and low dark current would result, since; in this case, the response speed of the MSM-PD is primarily limited by the transit time of the optically generated carriers and the RC-time constant of
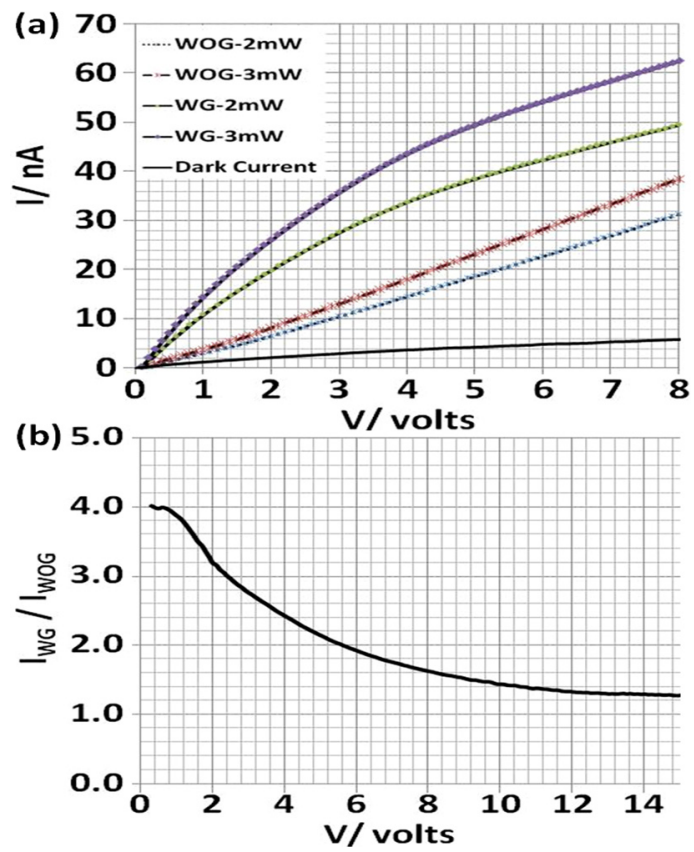

FIG. 4. (Color online) (a) Measured I-V characteristics comparing two GaAs MSM-PDs WGs and WOGs for different illumination power levels. (b) Photocurrent enhancement factor $\left(\mathrm{I}_{\mathrm{WG}} / \mathrm{I}_{\mathrm{WOG}}\right)$ versus input voltage for an input laser power of $3 \mathrm{~mW}$. the interdigitated PD structure. Note that etching multiple nano-size metal fingers results in a higher light power being absorbed in the semiconductor region. However, this results in a higher number of alternating thinner metal fingers that increase both the total capacitance and the total resistance of the MSM-PD, thus leading to a much lower speed as reported by Burm et al., ${ }^{16}$ and Yuang and Chyi. ${ }^{17}$ For a slit width in the order of $200 \mathrm{~nm}$, at low bias the semiconductor region of the MSM-PD is completely depleted and the electric field through the slit becomes high enough that the carriers drift at near saturation velocities. Under this condition, the transit time is simply determined by $\tau_{d r}=t \chi / 2 v$ as shown in Ref. 18, where $t$ is the gap width between the fingers, $v$ is carriers drift mean velocity, and $\chi$ is carrier drift distance corrective coefficient. Given the mean velocity of $6.7 \times 10^{6} \mathrm{~cm} / \mathrm{s}$ and the carrier drift distance corrective coefficient $\chi=1.3$ for the electron and hole in GaAs, the transit time of carrier and the frequency response bandwidth $\left(f_{t r}\right)$ are estimated to be 1.9 ps and $83 \mathrm{GHz}$, respectively.

In conclusion, we have designed and fabricated a plasmonics-based MSM-PD device employing metal nano-gratings and subwavelength slit. FDTD method has been used to optimize the various device parameters, and a dual beam FIB/ SEM has been employed for the fabrication of the metal nanogratings and slit of the MSM-PD. We have experimentally demonstrated the principle of plasmonics-based MSM-PDs and attained a measured photodetector responsivity that is 4-times better than that of conventional single-slit MSM-PDs.

${ }^{1}$ J. B. D. Soole and H. Schumacher, IEEE J. Quantum Electron. 27(3), 737 (1991).

${ }^{2}$ M. Y. Liu and S. Y. Chou, Appl. Phys. Lett. 66, 2673 (1995).

${ }^{3}$ S. Averine, O. Bondarenko, and R. Sachot, Solid-State Electron. 46, 2045 (2002).

${ }^{4}$ S. Y. Chou, Y. Liu, and P. B. Fischer, J. Vac. Sci. Technol. 9, 2920 (1991).

${ }^{5}$ T. W. Ebbesen, H. J. Lezec, H. F. Ghaemi, T. Thio, and P. A. Wolff, Nature 391, 667 (1998).

${ }^{6}$ L. Martín-Moreno, F. J. García-Vidal, H. J. Lezec, A. Degiron, and T. W. Ebbesen, Phys. Rev. Lett. 90, 167401 (2003).

${ }^{7}$ F. J. Garcia-Vidal, L. Martin-Moreno, T. W. Ebbesen, and L. Kuipers, Rev. Mod. Phys. 82, 729 (2010).

${ }^{8}$ T. Sondergaard, S. I. Bozhevolnyi, S. M. Novikov, J. Beermann, E. Devaux, and T. W. Ebbesen, Nano Lett. 10, 3123 (2010).

${ }^{9}$ J. A. Shackleford, R. Grote, M. Currie, J. E. Spanier, and B. Nabet, Appl. Phys. Lett. 94, 083501 (2009).

${ }^{10}$ G. Gay, O. Alloschery, B. V. d. Lesegno, J. Weiner, and H. J. Lezec, Phys. Rev. Lett. 96, 213901 (2006).

${ }^{11}$ J. A. Dionne, H. J. Lezec, and H. A. Atwater, Nano Lett. 6, 1928 (2006).

${ }^{12}$ S. Collin, P. Fabrice, R. Teissier, and J.-L. Pelouard, Appl. Phys. Lett. 85, 194 (2004).

${ }^{13}$ F. F. Ren, K. W. Ang, J. Ye, M. Yu, G. Q. Lo, and D. L. Kwong, Nano Lett. 11, 1289 (2011).

${ }^{14}$ S. C. Lee, S. Krishna, and S. R. J. Brueck, Appl. Phys. Lett. 97, 021112 (2010).

${ }^{15}$ N. Das, A. Karar, M. Vasiliev, C. L. Tan, K. Alameh, and Y. T. Lee, Opt. Commun. 284, 1694 (2011).

${ }^{16}$ J. Burm, K. I. Litvin, W. J. Schaff, and L. F. Eastman, IEEE Photon. Technol. Lett. 6(6), 722 (1994).

${ }^{17}$ R.-H. Yuang and J.-I. Chyi, Electron. Lett. 32(2), 131 (1996).

${ }^{18}$ S. Averine, Y. C. Chan, and Y. L. Lam, Solid-State Electron. 45, 441 (2001). 